\title{
METHOD FOR DETERMINING AMOUNT OF PRODUCT RELEASED INTO A TIME SENSITIVE OPERATION
}

\author{
Jonathan Levy \\ Richard Burda \\ Thomas Stahlecker \\ IBM Microelectronics \\ 2070 Route 52 \\ Hopewell Junction, NY, 12533, USA
}

\begin{abstract}
In manufacturing environments, there are potential requirements for a manufacturing step to be performed within a specific amount of time; failure to do so could result in product being defective or scrapped. This paper will show how a simulation model was used to define which factors are of utmost importance when determining the amount of product that can be released into one of these time sensitive steps.
\end{abstract}

\section{INTRODUCTION}

A time sensitive step (TSS) is defined as a manufacturing step which must be completed in a predetermined amount of time. For example, Figure 1 shows three steps in a generic manufacturing line.

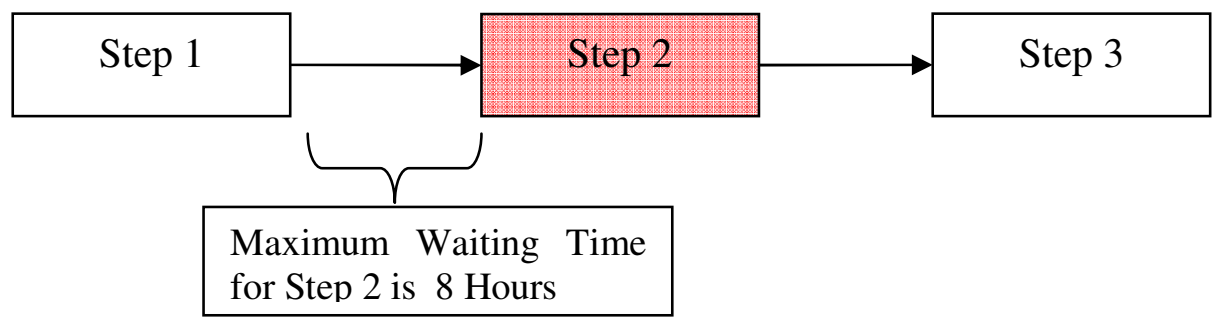

Figure 1: Step 2 is considered a time sensitive step.

According to Figure 1, product is in jeopardy for defects/scrap if it waits for more than 8 hours after being processed through Step 1. Step 1 is considered a trigger step. Step 3 is not a TSS since there is no limit as the amount of time product can wait before it is processed through Step 3. Additionally, Step 2 in Figure 1 often times represents a series of operations across several tool groups, all of which need to be completed within the time limit.

The challenges brought upon a manufacturing line by a TSS can be hard to overcome; in essence, one must estimate what is the optimal quantity of product waiting at a TSS. If the quantity is underestimated, then the TSS step will run idle before more product is processed in the trigger step. If the quantity is overestimated, then the probability of defects and scrap is increased.

Note that the risk of failing the TSS, that is the probability of product exceeding the duration of the TSS, will always be greater than zero. Also, the optimal quantity changes depending on the risk level. For 


\section{Levy, Burda and Stahlecker}

example, a TSS which is allowed to fail $10 \%$ of the time has a different optimal quantity than a similar TSS which is only allowed to fail $.01 \%$ of the time. In this paper, the tolerable risk level is set to be $1 \%$.

Another challenge that must be taken into consideration is that the optimal quantity of product waiting at a TSS is dynamic; it changes as the capacity of the TSS changes. This paper explores a simulation model which was built with the goal of determining which factors have the greatest effect on this optimal quantity. Based on previous manufacturing knowledge and experience the five factors that are going to be explored are:

- Time Sensitive Step Duration (amount of time product can wait before yield degrade)

- $\quad$ TSS Raw Processing Time (RPT)

- Number of like tools in the TSS

- Mean Time Between Fails (MTBF) for the tools in the TSS

- Mean Time Between Repairs (MTBR) for the tools TSS

This paper consists of the following sections:

- Section 2 provides background and reasons behind the model

- Section 3 provides an overview of the simulation model

- Section 4 describes the methodology used in this experiment.

- Section 5 details results

- Section 6 discusses conclusions

- Section 7 examines future work and additional consideration

\section{BACKGROUND}

The work described in this paper was done in support of the IBM $300 \mathrm{~mm}$ semiconductor fab in East Fishkill, NY (B323). B323 is similar to most $300 \mathrm{~mm}$ fabs in that there is a high level of automation in material handling as well as in the decision support that coordinates manufacturing activities. B323 is unique in the business role it serves and by consequence, is unique in the diversity of chips that are produced there.

B323 produces processors for IBM servers and is the semiconductor technology development fab for IBM and its development partners. B323 also acts as a chip foundry, producing chips for $3^{\text {rd }}$ party customers. Serving these three roles drives diversity in both production technologies and the product mix within the technologies. This diverse product mix shares a majority of the process tools in the fab.

The large product mix utilizing a shared resources within B323 makes management of TSS more complex than for fabs producing a more homogeneous product mix. B323 has thousands of TSS defined for WIP active on hundreds of process flows. Requirements were developed for an automated TSS WIP release system that could control WIP flow into TSS with a fab-wide view, considering all active TSS WIP and all WIP in queue at all TSS trigger operations.

Due to the wide penetration of TSS across process flows, the TSS WIP release system project posed significant risk to the operation of B323. If too little WIP is released into the TSS, the fab can experience a fab wide drop in tool utilization, reducing fab productivity, serviceability performance and delay technology development milestones. If too much WIP is released into TSS, fab rework will increase and product yield will degrade, again causing damages for all fab customers.

Indeed the automated TSS WIP release system project could not be approved without a method to predict the effects on tool and fab productivity and validate the control parameters introduced by the proposed system. A fab-wide discrete event simulation of B323 did not exist with the necessary process detail to be used for system design or testing. Lead time and cost constraints prevented building such a model in support of the TSS control project. 


\section{Levy, Burda and Stahlecker}

The project team aimed to test the system at a more generic level with a model that could be created quickly, considering all relevant TSS control parameters and accurately simulating the response of the true manufacturing system. This paper will describe how the team approached this challenge and how the resulting model was used to set the control parameters for the TSS WIP release system as it was introduced into production in $\mathrm{B} 323$.

\section{SIMULATION MODEL}

The simulation model was built using Arena 7.0. There are three routines that are processes in the model: the manufacturing routine, product release routine, and the artificial intelligence routine.

\subsection{Manufacturing Routine}

The manufacturing routine consists of product arriving into a trigger step as defined in figure 1. Product is held before processing at the trigger step until a released signal is issued by the product release routine. Once a release signal is issued, product is released to be processed by the trigger step, then processed through the time sensitive step, and finally it is decided if the product passed the time requirements. Figure 2 illustrates the manufacturing routine.

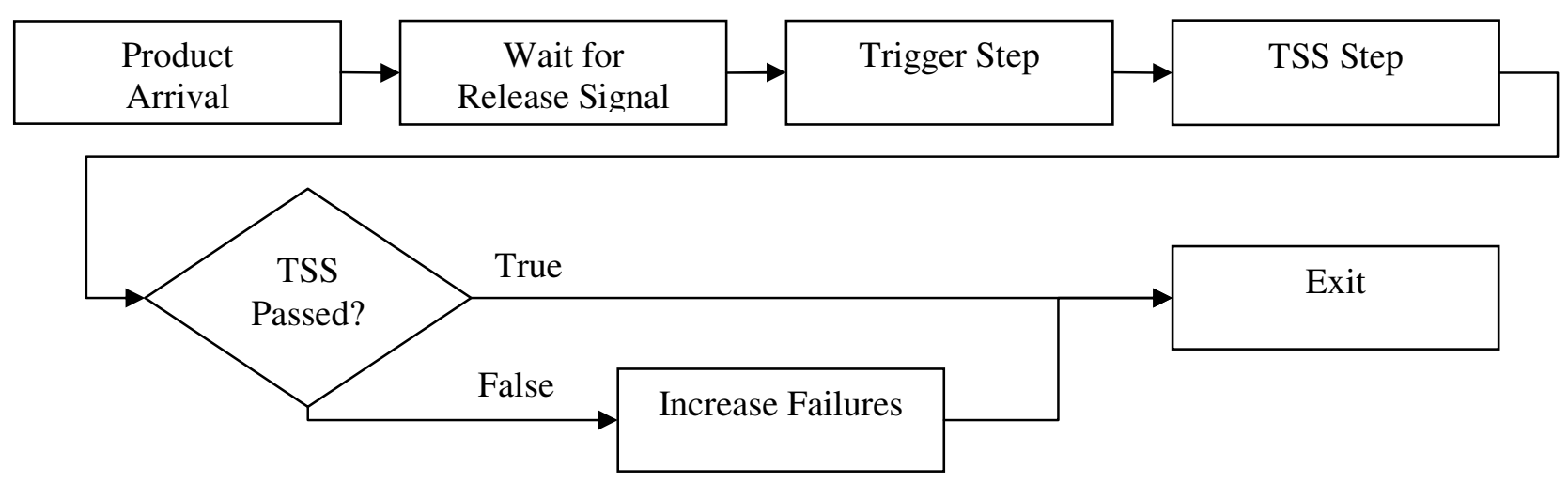

Figure 2: Manufacturing Routine

\subsection{Product Release Routine}

The product release routine is evaluated constantly and is responsible for issuing product release signals to the manufacturing routine. This is accomplished by comparing the capacity of the time sensitive step (TSSCapacity) to the product demand that has already been released through the trigger step (TSSDemand). If the capacity is greater than the demand, the manufacturing routine will release product into the trigger step as illustrated in Figure 3. Alternatively, if the capacity is smaller than the demand no instructions are sent to the manufacturing routine.

TSSCapacity is defined as the number of tools that are currently available at a particular step times a factor called PAT (Product Allowed per Tool); the PAT factor will be automatically calculated by the simulation model. Note that PAT is independent of demand, it is the maximum amount of product that can be released into the TSS.

TSSDemand is defined as the amount of product that is currently present at the TSS plus the amount of incoming product into that TSS. 


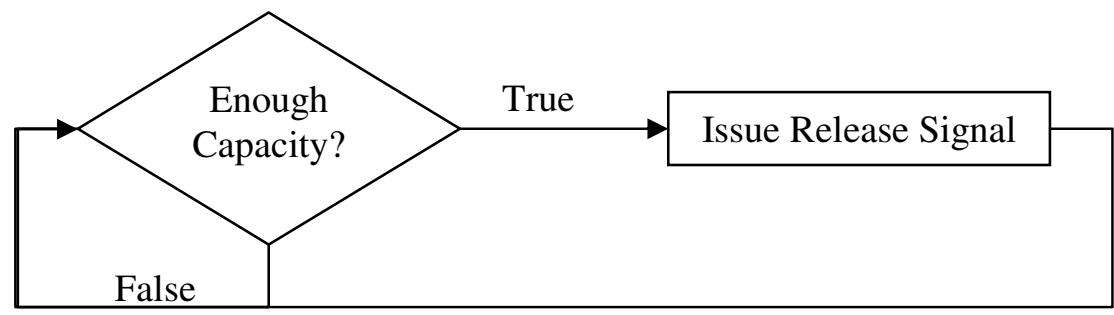

Figure 3: Product Release Routine

\subsection{Artificial Intelligence Routine}

The goal of the artificial intelligence routine is to automatically adjust PAT which is used to moderate how much product can be allowed into the TSS in the product release routine. PAT will increase if the failure $\%$ is greater than $1 \%$. Similarly, PAT will decrease if the failure $\%$ is less than $1 \%$. A representation of the Artificial Intelligence Routine is shown in Figure 4.

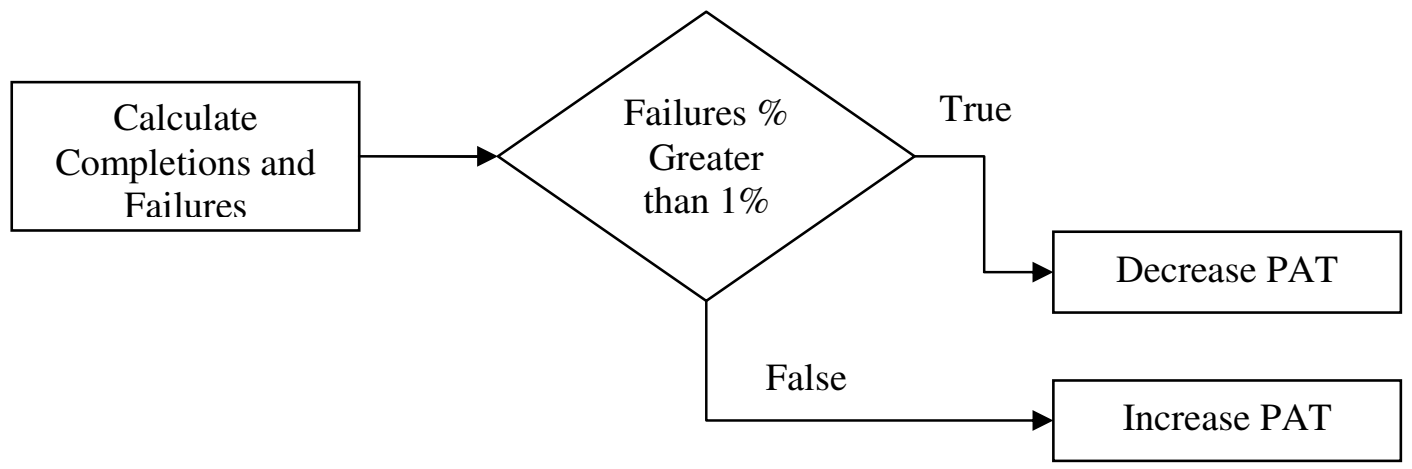

Figure 4: Artificial intelligence routine

\section{METHODOLOGY}

The experiment was setup with 3 factors, each factor had 3 levels as shown on the Table 1 . The tool availability was kept constant, therefore as the down time duration of the tools at the TSS changed, the time between fails increase/decreased accordingly.

Six simulation replications are run for each combination of factors for a tool for a total of 162 data points. Each replication length simulates 10,000 hours; the run length is set long in order to allow PAT to reach steady state. After each simulation, the PAT factor was recorded.

Table 1: Experiment factors

\begin{tabular}{|c|c|}
\hline Factor & Level \\
\hline \multirow{2}{*}{ TSS Raw Processing Time } & 30 minutes \\
\cline { 2 - 2 } & 2 hours and 45 minutes \\
\cline { 2 - 2 } \# of Tools at TSS Step & 5 hours \\
\cline { 2 - 2 } & 2 tools \\
\cline { 2 - 2 } & 5 tools \\
\hline \multirow{2}{*}{ Down Time Duration of Tools at TSS Step } & 8 tools \\
\cline { 2 - 2 } & 5 hours \\
\cline { 2 - 2 } & 22.5 hours \\
\hline
\end{tabular}




\section{Levy, Burda and Stahlecker}

Additionally, seven different TSS durations were chosen between the values of 3 to 20 hours. Each duration was replicated 6 times for an additional 42 data points. After each simulation, the PAT factor was recorded with the goal of understanding how PAT changes as the TSS duration changes.

\section{RESULTS}

The output from the simulations was analyzed using StatGraphics with Factorial Design analysis. Table 2 shows the data used that was collected after running the factorial design through the simulation model.

Table 2: Design of experiment results

\begin{tabular}{|c|c|c|c|}
\hline RPT & $\begin{array}{l}\text { Down Time } \\
\text { Duration }\end{array}$ & Tools & PAT \\
\hline 0.5 & 5 & 2 & 11.2 \\
\hline 2.75 & 5 & 2 & 1.4 \\
\hline 5 & 5 & 2 & 1.07 \\
\hline 0.5 & 22.5 & 2 & 3.7 \\
\hline 2.75 & 22.5 & 2 & 1.37 \\
\hline 5 & 22.5 & 2 & 1.04 \\
\hline 0.5 & 40 & 2 & 3.3 \\
\hline 2.75 & 40 & 2 & 1.5 \\
\hline 5 & 40 & 2 & 1.09 \\
\hline 0.5 & 5 & 5 & 22.9 \\
\hline 2.75 & 5 & 5 & 2.69 \\
\hline 5 & 5 & 5 & 1.28 \\
\hline 0.5 & 22.5 & 5 & 22.9 \\
\hline 2.75 & 22.5 & 5 & 2.87 \\
\hline 5 & 22.5 & 5 & 1.33 \\
\hline 0.5 & 40 & 5 & 23.7 \\
\hline 2.75 & 40 & 5 & 3.2 \\
\hline 5 & 40 & 5 & 1.35 \\
\hline 0.5 & 5 & 8 & 36.6 \\
\hline 2.75 & 5 & 8 & 3.4 \\
\hline 5 & 5 & 8 & 1.3 \\
\hline 0.5 & 22.5 & 8 & 30.5 \\
\hline 2.75 & 22.5 & 8 & 3.6 \\
\hline 5 & 22.5 & 8 & 1.4 \\
\hline 0.5 & 40 & 8 & 29.8 \\
\hline 2.75 & 40 & 8 & 3.8 \\
\hline 5 & 40 & 8 & 1.47 \\
\hline
\end{tabular}

Figure 5 shows a main effects plot based on the data shown on Table 2. The main effects plot shows how RPT and the number of tools available has a significant effect on the amount of product that can be released into a TSS. An increase in the MTBF and MTTR for a tool in a TSS does not have an effect on the amount of product that is allowed into the TSS.

As the RPT at a TSS increase the amount of product that can be allowed into the TSS decreases. As the number of tools at a TSS increase, the amount of product per tool that can be released into a TSS increases. 


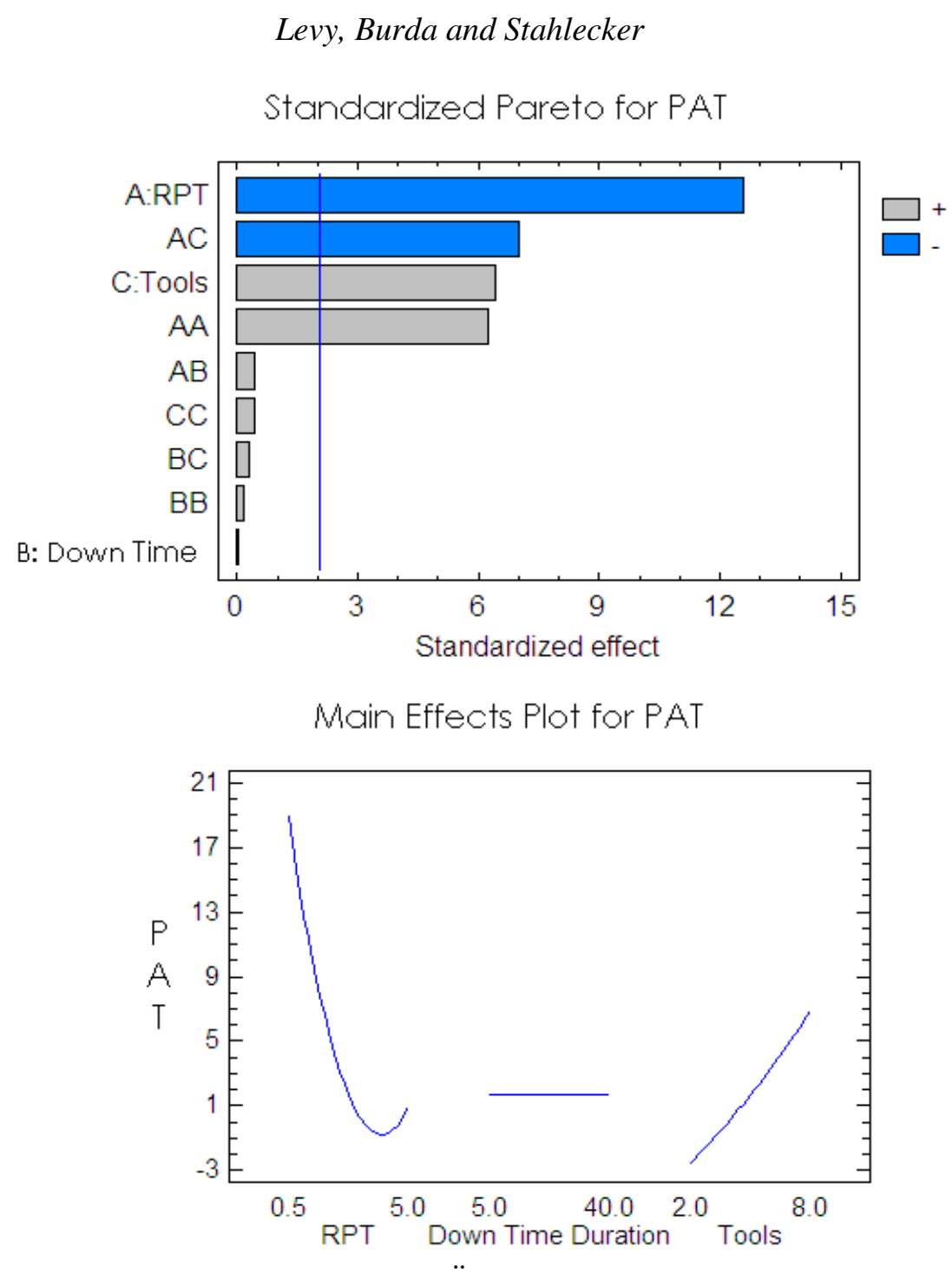

Figure 5: Main effects plot

Table 3 shows the data that was collected in order to understand how PAT changes as the TSS Duration increases.

Table 3: TSS Duration Sensitivity Analysis

\begin{tabular}{|c|c|c|}
\hline TSS Duration & PAT & PAT / TSS Duration \\
\hline 3 & 7.5 & 2.50 \\
\hline 5 & 12.5 & 2.50 \\
\hline 8 & 20 & 2.50 \\
\hline 10 & 25 & 2.50 \\
\hline 12 & 28 & 2.33 \\
\hline 15 & 32 & 2.13 \\
\hline 20 & 40 & 2.00 \\
\hline
\end{tabular}




\section{CONCLUSIONS}

The simulation model has illustrated how the raw processing time and the number of available tools has a significant impact on the amount of product per tool that can be released into a time sensitive step. Obviously as the RPT at a TSS increases, there is less capacity available and therefore the amount of product that is allowed into the TSS must decrease.

Based on the manufacturing operating curve, as the number of servers/tools increases the utilization of those tools can be increased without impacting the cycle-time of product. It is this property of the operating curve that justifies why the amount of product per tool that is released into a time sensitive step increases as the number of tools increases. One may also justify that result by reasoning that the probability of having all tools in an unavailable state when product arrives at a TSS decreases as the number of tools increases.

Surprisingly, an increase in the MTBF/MTTR of the tools in a time sensitive does not have an effect on the amount of product that can be released. This is believed to be to due to the fact that the simulation model only releases into the window based on tool availability. Therefore, the effect of an increase in MTBF is captured real time by the Product Release Routine.

As expected, the longer the duration of the TSS, the more product can be released into a TSS. This is due to the fact that more product is allowed to be queued in the TSS without increasing the probability of exceeding the TSS duration. There are diminishing returns on the PAT/ TSS Duration metric as Figure 6 shows. The simulation model result shows that for larger TSS durations there is no benefit of releasing more product when there is enough product at the TSS that those tools will not go idle.

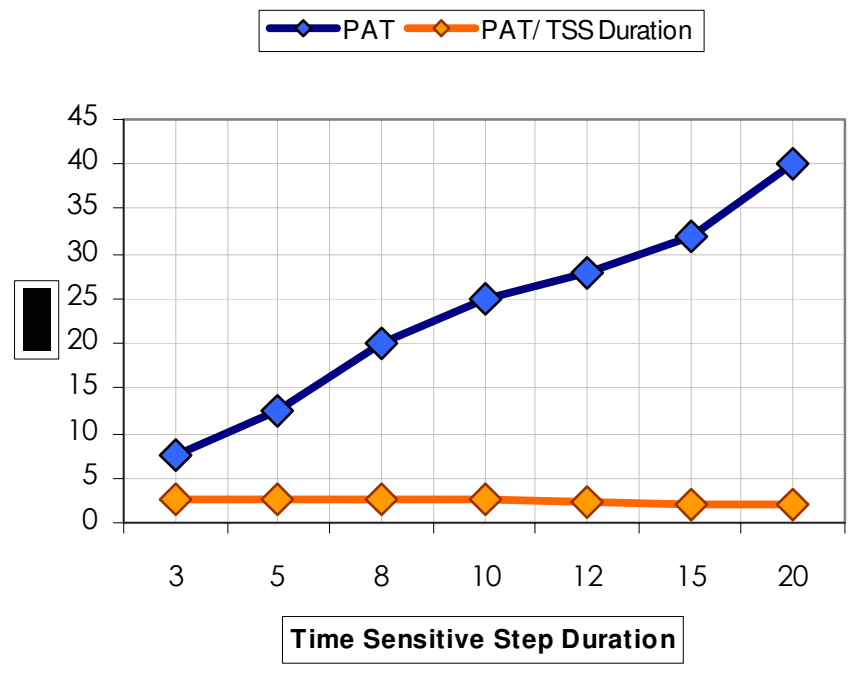

Figure 6: TSS Duration Sensitivity Charts

\section{FUTURE WORK}

This simulation only explored time sensitive steps that span one operation. In the future, a simulation model could be used to investigate the impacts of multi-step time requirements. For example, instead of having to complete one operation in 8 hours, three different operations have to be completed within 24 hours. Understanding the interactions between steps, and the effects the multi step time requirements are very hard to visualize, but could be discovered with a simulation model. 


\section{REFERENCES}

Gelenbe, E., and G. Pujolle. 1987. Introduction to queueing networks. New York: John Wiley \& Sons. Gross, D., and Harris C. 1985. Fundamentals of Queueing Theory: Second Edition,Wiley.

Hopp, W.J., and M.L. Spearman. 1996. Factory Physics: Foundations of Manufacturing Management, Burr Ridge, IL: Irwin/McGraw-Hill.

\section{AUTHOR BIOGRAPHIES}

JONATHAN LEVY is an Industrial Engineer for IBM's 300mm Semiconductor Fab in Fishkill, NY. He received his BS in Industrial and System Engineering from the University of Florida in Gainesville. His email address is <levyjona@us. ibm. com>.

RICH BURDA is the lead for 300mm scheduling and dispatch for IBM. Burda has held several positions relating to industrial engineering, operations management and product marketing in the semiconductor and other industries. Burda holds a BS in Mechanical Engineering from Villanova University and an MS in Management from the Pennsylvania State University. $\mathrm{He}$ can be contacted at <burda@us.ibm. com> .

THOMAS STAHLECKER is an Industrial Engineer for IBM's 300mm Semiconductor Fab in Fishkill, NY. He holds a BS in Industrial and Systems Engineering, and an MS in Applied Statistics from Rochester Institute of Technology. His email address is <tstahlec@us. ibm. com> . 